\title{
RADIO-QUIET AND RADIO-LOUD PULSARS: SIMILAR IN GAMMA-RAYS BUT DIFFERENT IN X-RAYS
}

\author{
M. Marelli ${ }^{1}$, R. P. Mignani ${ }^{1,2}$, A. De Luca ${ }^{1,3}$, P. M. Saz Parkinson ${ }^{4,5}$, D. Salvetti ${ }^{1}$, P. R. Den Hartog ${ }^{6}$, and M. T. WolfF ${ }^{7}$ \\ ${ }^{1}$ INAF-Istituto di Astrofisica Spaziale e Fisica Cosmica Milano, via E. Bassini 15, I-20133, Milano, Italy; marelli@iasf-milano.inaf.it \\ ${ }^{2}$ Kepler Institute of Astronomy, University of Zielona Góra, Lubuska 2, 65-265, Zielona Góra, Poland \\ 3 Istituto Nazionale di Fisica Nucleare, Sezione di Pavia, Via Bassi 6, I-27100 Pavia, Italy \\ ${ }^{4}$ Santa Cruz Institute for Particle Physics, Department of Physics, University of California at Santa Cruz, Santa Cruz, CA 95064, USA \\ 5 Department of Physics, The University of Hong Kong, Pokfulam Road, Hong Kong, China \\ ${ }^{6}$ Stanford University HEPL/KIPAC, 452 Lomita Mall, Stanford, CA 94305-4085, USA \\ ${ }^{7}$ Space Science Division, Naval Research Laboratory, Washington, DC 20375-5352, USA \\ Received 2014 November 19; accepted 2015 January 26; published 2015 March 24
}

\begin{abstract}
We present new Chandra and XMM-Newton observations of a sample of eight radio-quiet (RQ) $\gamma$-ray pulsars detected by the Fermi Large Area Telescope. For all eight pulsars we identify the X-ray counterpart, based on the $\mathrm{X}$-ray source localization and the best position obtained from $\gamma$-ray pulsar timing. For PSR J2030+4415 we found evidence for a $\sim 10^{\prime \prime}$-long pulsar wind nebula. Our new results consolidate the work from Marelli et al. and confirm that, on average, the $\gamma$-ray-to-X-ray flux ratios $\left(F_{\gamma} / F_{X}\right)$ of RQ pulsars are higher than for the radio-loud (RL) ones. Furthermore, while the $F_{\gamma} / F_{X}$ distribution features a single peak for the RQ pulsars, the distribution is more dispersed for the RL ones, possibly showing two peaks. We discuss possible implications of these different distributions based on current models for pulsar X-ray emission.
\end{abstract}

Key words: gamma rays: stars - pulsars: general - stars: neutron - X-rays: stars

\section{INTRODUCTION}

The launch of the Fermi $\gamma$-ray Space Telescope in 2008 marked a revolution in pulsar $\gamma$-ray astronomy (for a recent review see Caraveo 2014). The Large Area Telescope (LAT, Atwood et al. 2009) onboard Fermi has detected tens of millisecond and young-to-middle-aged rotation-powered $\gamma$-ray pulsars, with 117 listed in the Second Fermi LAT Catalog of $\gamma$ ray pulsars (2PC, Abdo et al. 2013). This number has already risen to more than $160 .{ }^{8}$ About $30 \%$ of the LAT pulsars are radio-quiet (RQ). In the $2 \mathrm{PC}, \mathrm{RQ}$ pulsars are defined as those that have not been detected in radio down to a flux density limit of $S_{1400}=30 \mu \mathrm{Jy}$ at $1400 \mathrm{MHz}$, whereas radio-loud (RL) pulsars are those detected above this limit. Some pulsars that have been detected in radio with a flux density below $S_{1400}=30 \mu \mathrm{Jy}$ are defined as radio faint (RF). Assuming that radio emission is produced close to the magnetic poles, this large fraction of RQ $\gamma$-ray pulsars suggests that the $\gamma$-ray beam is broader and at a larger angle from the magnetic poles with respect to the radio beam (see Caraveo 2014, and references therein), thus making its detection less sensitive to geometric effects. This piece of evidence has been crucial to verify the predictions of pulsar magnetospheric models, such as the outer gap and the polar gap ones (Cheng et al. 1986; Harding \& Muslimov 2004). Multi-wavelength studies of $\gamma$-ray pulsars are key to mapping the geometry of the different emission regions in the pulsar magnetosphere, investigating possible connections between different emission processes, and studying their efficiencies as a function of energy. For instance, Marelli et al. (2011) and Marelli (2012) showed that the distribution of the $\gamma$-to-X-ray flux ratio $F_{\gamma} / F_{\mathrm{X}}$ is different for RL and RQ pulsars, being narrower and peaking at higher values for the latter. What is behind the difference in the relative $\gamma$ and X-ray efficiencies of these two classes of $\gamma$-ray pulsars is not clear yet. While some clues are evident from the study of a handful of RQ pulsars detected in X-rays (see e.g. Marelli et al. 2013,

\footnotetext{
http://tinyurl.com/fermipulsars
}

2014a, 2014b),studying a larger sample allows one to explore differences across the parameter space. Furthermore, the detection of RQ $\gamma$-ray pulsars in X-rays (and in the optical) can provide information, for instance, on the pulsar dynamics and distance, traditionally obtained in the radio band. In fact, for RL pulsars the measurement of the dispersion of the pulses at different radio frequencies allows one to estimate the free electron column density, from which the distance to the pulsar is obtained from a gas distribution model (Cordes \& Lazio 2002). Alternatively, direct distance measurements for RL pulsars are obtained from the radio pulsar parallaxes. The measurement of the proper motion and distance of the RQ LAT pulsar PSR J0357+0352 with Chandra and XMM-Newton (De Luca et al. 2013; Marelli et al. 2013) is a spectacular example of distance estimation that is possible also for RQ pulsars and using a different method.

The X-ray satellites that have yielded the highest pulsar detection rates have traditionally been Chandra and XMMNewton due to their spatial, timing and spectral resolutions, along with their low background rates. Here, we present the results of new follow-up observations of a sample of RQ LAT pulsars with no previous X-ray detections. Our observations are summarized in Section 2, while our data analysis and results are described in Section 3. In Section 4 we compare the X-and $\gamma$-ray properties of all RQ LAT pulsars, making some considerations based on current models of $\gamma$-ray and X-ray emission from pulsars.

\section{TARGET SELECTION AND OBSERVATION DESCRIPTION}

We selected our target pulsars for Chandra and XMMNewton observations among those RQ LAT pulsars that have no, or uncertain, detection in X-rays (see Table 5 of $2 \mathrm{PC}$ ). In order to predict the non-thermal X-ray flux of these pulsars, we relied on the observed $F_{\gamma} / F_{\mathrm{X}}$ distribution of the RQ pulsar family, favoring pulsars with relatively high $\gamma$-ray flux and small pseudo-distance $D_{\gamma}$, as inferred from the comparison 
Table 1

Characteristics of the $\gamma$-ray Pulsars Discussed in this Work

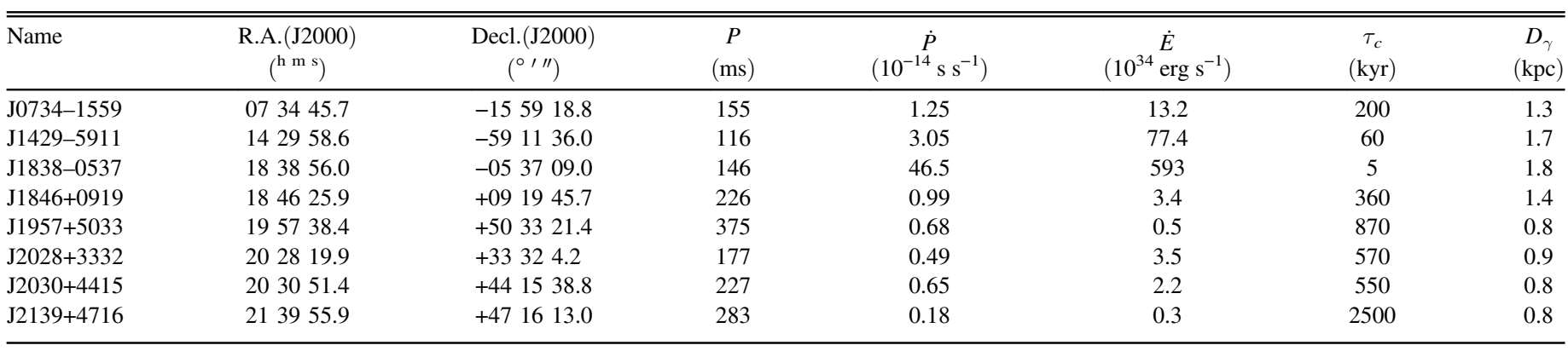

Note. Here, we list the name, $\gamma$-ray timing coordinates, period $(P)$, period derivative $(\dot{P})$, energetics $(\dot{E})$, characteristic age $\left((\tau)\right.$, and pseudo-distance $\left(D_{\gamma}\right)$, respectively. These values are taken from Abdo et al. (2013). Pulsars positions are taken from the last LAT $\gamma$-ray pulsar timing models, where possible, and from Pletsch et al. (2012a) for PSR J1838-0537.

between flux and luminosity of LAT pulsars with known distance (Saz Parkinson et al. 2010). Our sample includes eight LAT pulsars, spanning three orders of magnitude in characteristic ages $\left(\tau_{c} \equiv P / 2 \dot{P}\right.$, where $P$ is the neutron star spin period and $\dot{P}$ its derivative) and two orders of magnitude in spindown energies $\left(\dot{E} \equiv 4 \pi^{2} I \dot{P} / P^{3}\right.$, where the moment of inertia $I$ is assumed to be $10^{45} \mathrm{~g} \mathrm{~cm}^{2}$ ). Their $\gamma$-ray timing coordinates, which we used as a reference for the X-ray counterpart identification, and their timing parameters are summarized in Table 1, together with the values of the pseudo-distance $D_{\gamma}$, as defined in Saz Parkinson et al. (2010). We took the pulsar timing parameters and the pseudo-distance values from the compilations in Abdo et al. (2013), whereas we took the $\gamma$-ray timing positions and errors from the most recent compilation of the LAT $\gamma$-ray Pulsar Timing Models. ${ }^{9}$ This compilation reports the results of the analysis of five years of Fermi data by using a new, advanced timing analysis pipeline (M. Kerr et al. 2015 , in preparation) that handles glitch detection and fitting in the presence of timing noise much better than previous codes. We note that before this work, the positional errors based on $\gamma$ ray timing were correctly calculated (e.g., taking into account timing noise and systematic errors) only for a small number of bright pulsars (see e.g. Ray et al. 2011).

Swift already observed all the $\gamma$-ray pulsars in our sample in short $(\approx 5-10 \mathrm{ks})$ snapshot observations. Through the reanalyses of these data, we found no X-ray source that could be positionally associated with the pulsar (for a more detailed discussion see Marelli 2012). Three of these pulsars were also re-observed by Suzaku: two of them (PSR J1429-5911 and $\mathrm{J} 1957+5033)$ remained undetected, also owing to the short exposure times, whereas a possible, marginal X-ray detection was obtained for PSR J1838-0537.

Here, we report on five new $25 \mathrm{ks}$ Chandra observations of PSRs J1429-5911，J1957+5033，J2028+3332，J2030+4415, and J2139+4716 (observation ids 14825, 14828, 14826, 14827 and 14829 , respectively) and 15 ks new Chandra observations of PSRs J0734-1559 and J1846+0919 (observation ids 13792 and 13793, respectively). We observed all the pulsars with the ACIS. In order to detect the highest number of counts from our targets we obtained the ACIS-S observations in the VFAINT mode, with the targets' positions placed on the backilluminated ACIS S3 chip. We observed PSR J1838-0537 with XMM-Newton for 44 ks (obs. id 0720750201) using The

\footnotetext{
9 https://confluence.slac.stanford.edu/display/GLAMCOG/LAT+Gamma-ray + Pulsar+Timing+Models
}

EPIC (European Photon Imaging Camera) PN and the two metal oxide semi-conductor (MOS) cameras in the Full Frame mode with medium optical filters, due to the presence of moderately bright stars within their field of view. All the observations were carried out between 2012 September 16 and 2014 April 15.

\section{DATA ANALYSIS AND RESULTS}

For the Chandra data analysis we used the Chandra Interactive Analysis of Observation (CIAO) software version 4.3. We re-calibrated our event data by using the chandra_repro script and selected events in the $0.3-10 \mathrm{keV}$ energy range. Depending on the total number of counts, we ran the wavdetect detection tool in different energy ranges, taking into account the exposure maps. For each pulsar, we found a single X-ray source within (or close to) the $95 \% \gamma$-ray timing error box, with a detection significance above $5 \sigma$ and source counts varying from 12 (PSR J1846+0919) to 123 (PSR J1957+5033). For each X-ray source we performed a spectral analysis. We extracted the source counts from a $2^{\prime \prime}$ circle radius around the X-ray position and the background counts from a surrounding annulus, whose radius we chose on a case by case basis in order to avoid contamination from serendipitous nearby sources. We used the CIAO tool specextract to create the spectra, response matrix and effective area files and analyzed the spectra with XSPEC (version 12.8.1). We used the C-statistic (Cash 1979), based on the application of the likelihood ratio and recommended for cases with low statistics and low background. As done in Marelli et al. (2013), we performed a brightness profile analysis on all X-ray sources, revealing no evidence for extended emission, with the exception of PSR J2030+4415 (see Section 3.7).

For the analysis of the $44 \mathrm{ks}$ XMM observation of PSR J1838-0537, we used the XMM-Newton Science Analysis Software (SAS) v13.0. We performed a standard analysis of high particle background (following De Luca et al. 2005) and cross-checked the results with the SAS tool bkgoptrate (also used for the $3 \mathrm{XMM}$ source catalog ${ }^{10}$ ), obtaining very good agreement. After the subtraction of bad time intervals affected by soft proton X-ray flares, we obtained a net exposure time of $23 \mathrm{ks}$. We selected $0-4$ pattern events for PN and 0-12

\footnotetext{
$\overline{10}$ http://xmmssc-www.star.le.ac.uk/Catalogue/xcat_public_3XMMDR4.html
} 
for the MOS detectors in the $0.3-10 \mathrm{keV}$ energy range. From the cleaned events, we ran the source detection according to two different methods: we used the SAS edetect-chain and the CIAO wavdetect tools. Both of them detected a single X-ray source within a few arcsecs from the pulsar $\gamma$-ray timing position. The source appears point-like, with its brightness profile consistent with the detector's point spread function. In order to maximize the source signal-to-noise ratio, we extracted spectral counts from a $25^{\prime \prime}$ radius circular region around the computed X-ray position and the background from a source-free, nearby, $40^{\prime \prime}$ radius circle on the same CCD. We generated ad hoc response matrices and effective area files using the SAS tools rmfgen and arfgen. To increase the statistics, we added the two MOS spectra by using the HeaSoft tool mathpha and the two response matrices and effective area with addrmf and addarf. After background subtraction, we extracted 200 and 176 net source counts from the PN and the two MOS, respectively. Owing to the high background (64\% and $43 \%$ of the extracted counts from the PN and MOS, respectively), we used the $\chi^{2}$ statistic for the spectral fit.

Due to the low statistics of our X-ray sources, we assumed the pulsar pseudo-distance $D_{\gamma}$ to normalize the value of the integrated Galactic $N_{H}$ in the pulsar direction, computed according to the recalibration (Schlafly \& Finkbeiner 2011) of the extinction maps from Schlegel et al. (1998). Four of our eight sources have fewer than 20 net counts, which are not adequate to perform detailed spectral fits. Therefore, for these sources we fixed the power-law (PL) photon index and blackbody $(\mathrm{BB})$ temperature to representative values of 2 and $200 \mathrm{eV}$, respectively. Following, e.g., Marelli et al. (2011), these are the fitted average of the values measured for pulsars detected in the X-rays. Errors in the spectral parameters are reported at the $90 \%$ confidence level (c.l.).

The errors on the X-ray positions reported in the following sub-sections are purely statistical, at a $3 \sigma$ confidence. To them, we have to add the $90 \%$ c.l. systematic errors associated with the absolute accuracy of the satellite aspect solution, which are $0 ! .8$ and 1'.5 per coordinate for Chandra ${ }^{11}$ and XMM-Newton, ${ }^{12}$ respectively. According to the $\log N-\log S$ distribution of Chandra Galactic sources (Novara et al. 2009), we can estimate the probability of a chance detection of an X-ray source within a representative LAT $\gamma$-ray timing error box $\left(1^{\prime \prime 2}\right.$, see Figure 1$)$, with X-ray flux similar to or greater than the measured ones, to be about $3 \times 10^{-5}$. Thus, based on positional coincidence, we consider our identifications to be secure. Figure 1 shows the positions and errors of $\gamma$-ray pulsars and the associated X-ray counterparts. The computed X-ray spectral parameters, unabsorbed X-ray fluxes, and $\gamma$-to-X-ray flux ratios of our eight pulsars are summarized in Table 2.

\subsection{PSR J0734-1559}

PSR J0734-1559 ( $P=156 \mathrm{~ms})$ was identified as a middleaged $(0.2 \mathrm{Myr}) \gamma$-ray pulsar during a blind search for pulsations from the unidentified LAT source 1FGL J0734.7-1557 (Saz Parkinson 2011). We detected its X-ray counterpart at $\alpha_{\mathrm{X}}=07^{\mathrm{h}} 34^{\mathrm{m}} 45^{\mathrm{s}} .7( \pm 0.15) ; \delta_{\mathrm{X}}=-15^{\circ} 59^{\prime} 19^{\prime \prime} .8( \pm 0.26)$, consistent with the pulsar $\gamma$-ray timing coordinates. The X-ray source is detected with a significance of $8.3 \sigma$, as computed by wavdetect, with 19 net counts. As described above, we

\footnotetext{
$11 \mathrm{http} / / / \mathrm{cxc}$.harvard.edu/cal/ASPECT/celmon/

12 Calibration technical note XMM-SOC-CAL-TN-0018.
}

assumed the pulsar pseudo-distance $D_{\gamma}=1.3 \mathrm{kpc}$ to estimate a $N_{H}=2 \times 10^{21} \mathrm{~cm}^{-2}$. Keeping these values fixed, we fitted the $\mathrm{X}$-ray spectrum of the pulsar with either a single PL or BB model. Due to the low statistics, we also fixed the PL photon index to 2 and the BB temperature to $200 \mathrm{eV}$. The best-fit with a $\mathrm{BB}$ gives a radius of the emitting region on the neutron star surface of $76_{-11}^{+16} \mathrm{~m}$, computed for the assumed pseudo-distance $D_{\gamma}$. For the PL model, the unabsorbed X-ray flux in the $0.3-10 \mathrm{keV}$ energy range is $F_{\mathrm{X}}^{\mathrm{PL}}=1.5 \pm 0.6 \times 10^{-14}$ erg $\mathrm{cm}^{-2} \mathrm{~s}^{-1}$, which gives a $\gamma$-to-X-ray flux ratio $F_{\gamma} / F_{\mathrm{X}} \sim 3700$. The $\mathrm{BB}$ model, on the other hand, gives an $\mathrm{X}$-ray flux $F_{\mathrm{X}}^{\mathrm{BB}}=1.2 \pm 0.4 \times 10^{-14} \mathrm{erg} \mathrm{cm}^{-2} \mathrm{~s}^{-1}$ and an $F_{\gamma} / F_{\mathrm{X}}>4700$, assuming $F_{\mathrm{X}}^{\mathrm{BB}}$ as an upper limit on the nonthermal X-ray flux from the pulsar.

\subsection{PSR J1429-5911}

The $\gamma$-ray pulsar PSR J1429-5911 $(P=115 \mathrm{~ms})$ was one of the very first discovered by applying the blind-search technique (Saz Parkinson et al. 2010). We detected its X-ray counterpart at $\alpha_{\mathrm{X}}=14^{\mathrm{h}} 29^{\mathrm{m}} 58.5\left( \pm 1^{\prime \prime} \cdot 03\right) ; \delta_{\mathrm{X}}=-59^{\circ} 11^{\prime} 36^{\prime \prime} \cdot 2( \pm 0.45)$, with a significance of $8.5 \sigma$ (24 net source counts). As we did in the previous section, we assumed the pseudo-distance $D_{\gamma}$ $=1.7 \mathrm{kpc}$ to estimate an $N_{\mathrm{H}}=3 \times 10^{21} \mathrm{~cm}^{-2}$ and we kept it fixed in the X-ray spectral fit. In this way, a fit with a PL gives a photon index $\Gamma_{\mathrm{X}}=-0.1 \pm 0.7$ and an unabsorbed X-ray flux $F_{\mathrm{X}}^{\mathrm{PL}}=(3.3 \pm 1.8) \times 10^{-14} \mathrm{erg} \mathrm{cm}^{-2} \mathrm{~s}^{-1}$. This corresponds to $F_{\gamma} / F_{\mathrm{X}} \sim 2400$. A fit to the data with a single $\mathrm{BB}$ model did not yield an acceptable temperature $(T>0.8 \mathrm{keV})$.

\subsection{PSR J1838-0537}

The $\gamma$-ray pulsar $(P=145 \mathrm{~ms})$ PSR J1838-0537 was discovered through a blind search for pulsations of the unassociated LAT source 2FGL J1839.0-0539 (Pletsch et al. 2012a). The pulsar is the youngest ( $5 \mathrm{kyr}$ ) and most energetic $\left(\dot{E} \sim 5.9 \times 10^{36} \mathrm{erg} \mathrm{cm}^{-2} \mathrm{~s}^{-1}\right)$ in our sample. PSR J1838 -0537 is spatially coincident with the TeV source HESS J1841 -055 , hence possibly associated with a pulsar wind nebula (PWN) detected at very high energies. A $41.1 \mathrm{ks}$ Suzaku observation revealed a candidate X-ray counterpart (Pletsch et al. 2012a). However, the non-negligible probability of a finding a spurious source within the large Suzaku error circle $\left(\approx 19^{\prime \prime}\right.$ radius, Uchiyama et al. 2008), as well as the low significance of detection $(\sim 3 \sigma)$ made the identification of the $\mathrm{X}$-ray source with the pulsar uncertain. Owing to the better angular resolution of XMM-Newton we detected the pulsar Xray counterpart at $\alpha_{\mathrm{X}}=18^{\mathrm{h}} 38^{\mathrm{m}} 56^{\mathrm{s}} .2 \quad\left( \pm 2{ }^{\prime \prime} \cdot 65\right)$; $\delta_{\mathrm{X}}=-05^{\circ} 37^{\prime} 04^{\prime \prime} 5\left( \pm 22^{\prime \prime} 69\right)$, with a significance of $20.7 \sigma$ ( $\sim 270$ counts; pn+MOS). The X-ray spectrum is best fitted by an absorbed PL with a photon index $\Gamma_{\mathrm{X}}=0.8_{-0.9}^{+1.1}$ and a fitted $N_{\mathrm{H}}=2.7_{-2.1}^{+4.4} \times 10^{22} \mathrm{~cm}^{-2}$ (90\% c.l.). The unabsorbed X-ray flux is $F_{\mathrm{X}}^{\mathrm{PL}}=(7.2 \pm 0.9) \times 10^{-14} \mathrm{erg} \mathrm{cm}^{-2} \mathrm{~s}^{-1}$, which gives a $\gamma$-to-X-ray flux ratio $F_{\gamma} / F_{\mathrm{X}} \sim 2600$. A fit with a BB spectrum did not yield an acceptable temperature $(T>1.5 \mathrm{keV})$.

\subsection{PSR J1846+0919}

PSR J1846+0919 $(P=225 \mathrm{~ms})$ was identified as a $\gamma$-ray pulsar by Saz Parkinson et al. (2010). We detected the X-ray counterpart at $\alpha_{\mathrm{X}}=18^{\mathrm{h}} 46^{\mathrm{m}} 25^{\mathrm{s}} .8( \pm 0.41) ; \delta_{\mathrm{X}}=09^{\circ} 19^{\prime} 49^{\prime \prime} .8$ $( \pm 0.45)$ with a significance of $5.4 \sigma$ (12 net source counts). As 

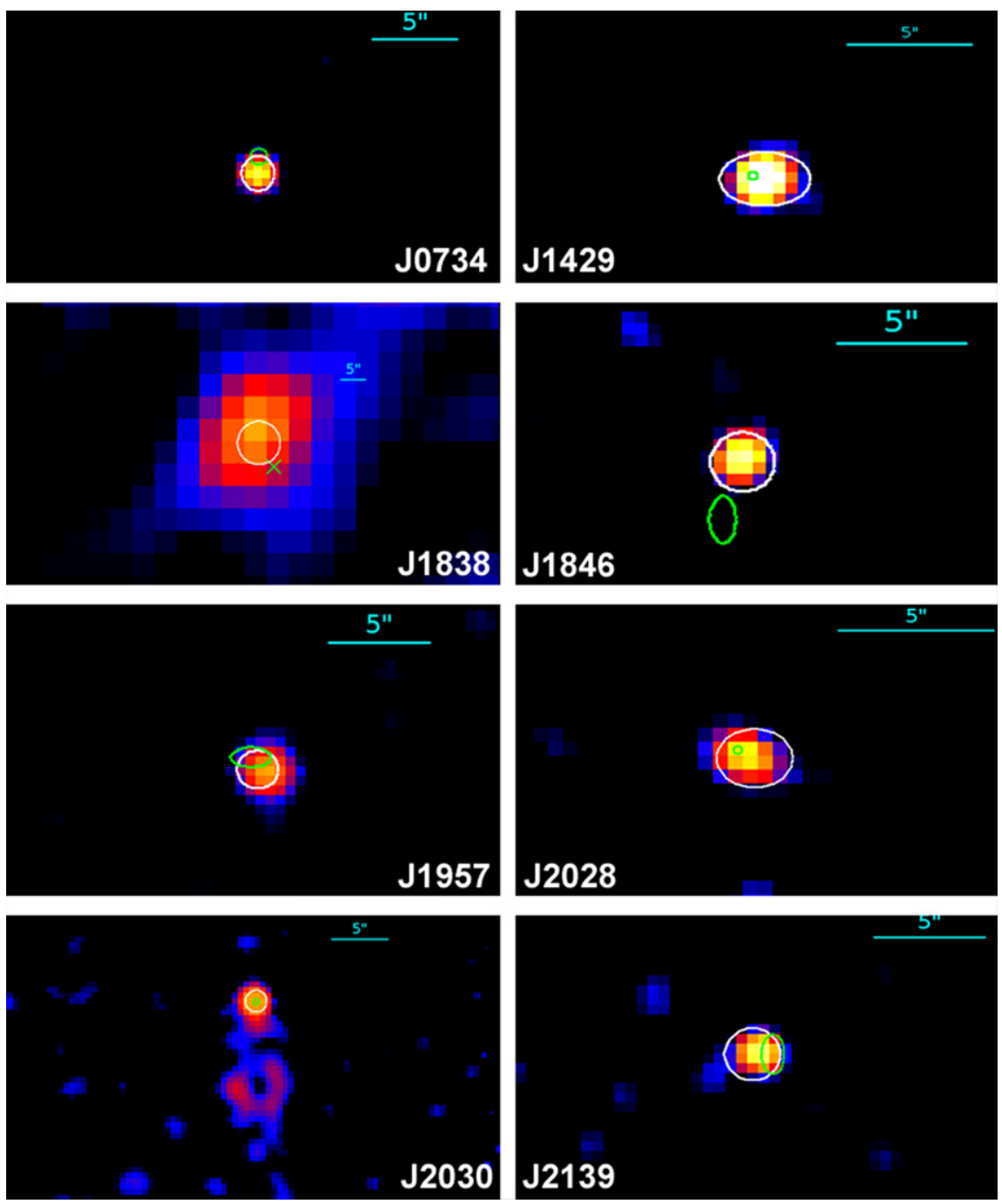

Figure 1. Chandra and XMM-Newton images of the pulsars listed in Table 1, taken in the $0.3-10 \mathrm{keV}$ energy range. For a better visualization, we applied a Gaussian filter with a kernel radius of $3^{\prime \prime}$. In each panel, the best-fit X-ray position ( $90 \%$ confidence errors) is shown by the white circle and the LAT $\gamma$-ray timing position ( $1 \sigma$ errors) by the green ellipses. The latter are taken from the most recent compilation of the LAT $\gamma$-ray Pulsar Timing Models. The position of PSR J1838-0537 is taken from Pletsch et al. (2012a), where the positional error is not reported, and is marked by the green cross.

Table 2

Summary of the X-ray Spectral Parameters for the Pulsars Discussed in this Work, as Described in Section 3

\begin{tabular}{|c|c|c|c|c|c|c|c|c|}
\hline Name & $\begin{array}{c}N_{H} \\
\left(10^{21} \mathrm{~cm}^{-2}\right)\end{array}$ & $\Gamma_{\mathrm{X}}$ & $\begin{array}{c}F_{\mathrm{X}}^{\mathrm{PL}} \\
\left(10^{-14} \mathrm{erg} \mathrm{cm}^{-2} \mathrm{~s}^{-1}\right)\end{array}$ & $F_{\gamma} / F_{\mathrm{X}}$ & $\begin{array}{c}k T \\
(\mathrm{eV})\end{array}$ & $\begin{array}{c}R \\
(\mathrm{~m})\end{array}$ & $\begin{array}{c}F_{\mathrm{X}}^{\mathrm{BB}} \\
\left(10^{-14} \mathrm{erg} \mathrm{cm}^{-2} \mathrm{~s}^{-1}\right)\end{array}$ & $F_{\gamma} / F_{\mathrm{X}}$ \\
\hline J0734-1559 & 2 & 2 & $1.5 \pm 0.6$ & $3700 \pm 800$ & 200 & $76_{-11}^{+16}$ & $1.2 \pm 0.4$ & $>4700$ \\
\hline J1429-5911 & 3 & $-0.1 \pm 0.7$ & $3.3 \pm 1.8$ & $2400 \pm 400$ & $\cdots$ & $\cdots$ & $\ldots$ & $\cdots$ \\
\hline J1838-0537 & $27_{-21}^{+44}$ & $0.8_{-0.9}^{+1.1}$ & $7.2 \pm 0.9$ & $2600 \pm 200$ & $\cdots$ & $\cdots$ & $\cdots$ & $\cdots$ \\
\hline $\mathrm{J} 1846+0919$ & 2 & 2 & $0.9 \pm 0.2$ & $2800 \pm 700$ & 200 & $45_{-13}^{+12}$ & $0.5 \pm 0.3$ & $>5300$ \\
\hline $\mathrm{J} 1957+5033$ & $<0.25$ & $2.1 \pm 0.3$ & $3.0 \pm 0.5$ & $870 \pm 70$ & $\cdots$ & $\cdots$ & $\ldots$ & $\cdots$ \\
\hline $\mathrm{J} 2028+3332$ & 2 & 2 & $0.5 \pm 0.3$ & $10900 \pm 4700$ & 200 & $34_{-8}^{+12}$ & $0.2 \pm 0.1$ & $>26400$ \\
\hline $\mathrm{J} 2030+4415$ & $0.61_{-0.61}^{+1.54}$ & $2.4_{-0.6}^{+0.8}$ & $2.1 \pm 0.6$ & $2800 \pm 400$ & $\cdots$ & $\cdots$ & $\cdots$ & $\cdots$ \\
\hline $\mathrm{J} 2139+4716$ & 1 & 2 & $0.5 \pm 0.3$ & $4900 \pm 1300$ & 200 & $33 \pm 9$ & $0.3 \pm 0.1$ & $>8500$ \\
\hline
\end{tabular}

Note. Here, we list the name, best fit column density, photon index, flux and gamma-to-X flux ratio in the case of non-thermal emission and temperature, radius, flux and lower limit gamma-to-X flux ratio in case of thermal emission, where acceptable. All quoted errors are at the $90 \%$ c.l., with the exception of the $F_{\gamma} / F_{\mathrm{X}}$ for which the $1 \sigma$ error is reported.

usual, from the pseudo-distance we estimated an $N_{\mathrm{H}}=2 \times 10^{21} \mathrm{~cm}^{-2}$ and kept it fixed in the X-ray spectral fit. A fit with a PL with fixed photon index $\left(\Gamma_{X}=2\right)$ gives an unabsorbed X-ray flux $F_{\mathrm{X}}^{\mathrm{PL}}=(8.7 \pm 4.8) \times 10^{-15} \mathrm{erg} \mathrm{cm}^{-2}$ $\mathrm{s}^{-1}$, which corresponds to $F_{\gamma} / F_{\mathrm{X}} \sim 2800$. Owing to the low statistics, the X-ray spectrum of this source can be fitted also by a BB. From the pseudo-distance of $1.4 \mathrm{kpc}$ we obtained an emitting radius of $75_{-18}^{+15} \mathrm{~m}$ and the unabsorbed flux is 
$F_{\mathrm{X}}^{\mathrm{BB}}=(4.5 \pm 3.3) \times 10^{-15} \mathrm{erg} \mathrm{cm}^{-2} \mathrm{~s}^{-1}$. Assuming, as before, $F_{\mathrm{X}}^{\mathrm{BB}}$ as an upper limit to the non-thermal X-ray flux of the pulsar, the lower limit on $F_{\gamma} / F_{\mathrm{X}}$ would be 5300 .

\subsection{PSR J1957+5033}

Like PSR J1429-5911 and J1846+0919, PSR J1957+5033 $(P=374 \mathrm{~ms})$ is one of the very first pulsars discovered by a blind search by Saz Parkinson et al. (2010). We detected the X-ray counterpart at $\alpha_{\mathrm{X}}=19^{\mathrm{h}} 57^{\mathrm{m}} 38^{\mathrm{s}} .4 \quad( \pm 0.20)$; $\delta_{\mathrm{X}}=50^{\circ} 33^{\prime} 20^{\prime \prime} 8\left( \pm 0^{\prime \prime} 17\right)$. The pulsar X-ray counterpart is detected with a significance of $29.0 \sigma$ (123 net source counts). Owing to the adequate statistics, we left the absorption column as a free parameter in our spectral fits, as in the case of PSR J1838-0537 (Section 3.3). The X-ray spectrum is best fit by a PL, which gives an $N_{\mathrm{H}}<2.5 \times 10^{20} \mathrm{~cm}^{-2}$, a photon index $\quad \Gamma_{X}=2.1 \pm 0.3$, and an unabsorbed flux

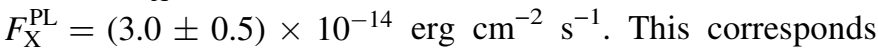
to a quite low $F_{\gamma} / F_{\mathrm{X}} \sim 870$, indeed the lowest among the pulsars in our sample. A fit with a single BB component is not acceptable (null hypotesis probability of $4 \times 10^{-8}$ ).

\subsection{PSR J2028+3332}

This pulsar $(P=176 \mathrm{~ms})$ is one of the nine $\gamma$-ray pulsars discovered by Pletsch et al. (2012b) using a novel blind search technique. We identified the PSR J2028+3332 X-ray counterpart at $\alpha_{\mathrm{X}}=20^{\mathrm{h}} 28^{\mathrm{m}} 19.8( \pm 0.45) ; \delta_{\mathrm{X}}=33^{\circ} 32^{\prime} 04^{\prime \prime} .^{\prime \prime}( \pm 0.23)$ with a significance of $5.1 \sigma$ ( 15 net source counts). As we did in previous cases, we used the pseudo-distance $\mathrm{D}_{\gamma}=0.9 \mathrm{kpc}$ to derive an $N_{\mathrm{H}}=2 \times 10^{21} \mathrm{~cm}^{-2}$. The fit with a PL with fixed photon index $\left(\Gamma_{X}=2\right)$ gives an unabsorbed $X$-ray flux $F_{\mathrm{X}}^{\mathrm{PL}}=(5.3 \pm 3.3) \times 10^{-15} \mathrm{erg} \mathrm{cm}^{-2} \mathrm{~s}^{-1}$, and $F_{\gamma} / F_{\mathrm{X}} \sim 10900$, the highest among the pulsars in our sample. We also tried a fit with a BB, which gives an emitting radius of only $34_{-8}^{+12} \mathrm{~m}$, an $\mathrm{X}$-ray flux $F_{\mathrm{X}}^{\mathrm{BB}}=(2.2 \pm 1.5) \times 10^{-15} \mathrm{erg} \mathrm{cm}^{-2} \mathrm{~s}^{-1}$, and an $F_{\gamma} / F_{\mathrm{X}}>26400$.

\subsection{PSR J2030+4415}

The $\gamma$-ray pulsar $\mathrm{J} 2030+4415(P=227 \mathrm{~ms})$ was also discovered by Pletsch et al. (2012b). The pulsar X-ray counterpart, at $\alpha_{\mathrm{X}}=20^{\mathrm{h}} 30^{\mathrm{m}} 51^{\mathrm{s}} .4( \pm 0.15) ; \delta_{\mathrm{X}}=44^{\circ} 15^{\prime \prime} 38^{\prime \prime} .8( \pm 0.16)$, is detected with a significance of $15 \sigma$ (54 net source counts). The $0.3-10 \mathrm{keV}$ X-ray spectrum is described by a PL with photon index $\Gamma_{\mathrm{X}, \mathrm{PSR}}=2.4_{-0.6}^{+0.8}$, for an $N_{\mathrm{H}}=6.1_{-6.1}^{15.4} \times 10^{20} \mathrm{~cm}^{-2}$, which gives an unabsorbed X-ray flux $F_{\mathrm{X}, \mathrm{PSR}}=$ $(2.1 \pm 0.6) \times 10^{-14} \mathrm{erg} \mathrm{cm}^{-2} \mathrm{~s}^{-1}$. The low value of the absorption column $\left(N_{\mathrm{H}} \lesssim 3 \times 10^{21} \mathrm{~cm}^{-2}\right)$ with respect to the Galactic $N_{\mathrm{H}}$ in the pulsar direction $\left(\sim 10^{22} \mathrm{~cm}^{-2}\right)$ agrees with the pseudo-distance $D_{\gamma}=800 \mathrm{pc}$. The $\gamma$-to-X-ray flux ratio for PSR $\mathrm{J} 2030+4415$ is $F_{\gamma} / F_{\mathrm{X}} \sim 2800$. A fit with a single BB component is not acceptable (null hypothesis probability of $6 \times 10^{-9}$ ). We found a $\sim 10^{\prime \prime}$ long extended emission around the pulsar, elongated North to South. To search for extended emission, we applied the CIAO tool vtpdetect on an event list purged from the point-like sources we found in the field using wavdetect. This tool, based on the Voronoi Tessellation and Percolation (VTP, see e.g. Boschin 2002), is particularly indicated for the search of extended sources. This resulted in a false source probability (i.e., the probability that the detection is associated to a real source) for PSR J2030+4415 nebula of $10^{-26}$ and a best- fitting elliptical region with a major axis of $\sim 10^{\prime \prime}$. Assuming the pseudo-distance of $800 \mathrm{pc}$, the "tail" would have a physical dimension of $\sim 0.07$ pc. Since PSR J2030+4415 is RQ and Chandra has observed it only once, we have no proper motion information yet, thus we cannot say whether the X-ray nebula is aligned with the motion of the pulsar, although this would be the most likely interpretation. We extracted the net counts from the PWN from an elliptical region of $10^{\prime \prime}$ semimajor axis after masking the pulsar. We computed the background from nearby, source-free elliptical regions. The tail spectrum is well described by a PL, with $\Gamma_{\mathrm{X}, \mathrm{PWN}}=1.2_{-0.4}^{+0.5}$, with the unabsorbed X-ray flux of $F_{\mathrm{X}, \mathrm{PWN}}=(4.0 \pm 1.6) \times 10^{-14} \mathrm{erg} \mathrm{cm}^{-2} \mathrm{~s}^{-1}$, about twice as large as the pulsar.

\subsection{PSR J2139+4716}

This $\gamma$-ray pulsar $(P=282 \mathrm{~ms}$; Pletsch et al. $2012 \mathrm{~b})$ is the oldest $(2.5 \mathrm{Myr})$ and least energetic $\left(\dot{E} \sim 0.3 \times 10^{34} \mathrm{erg} \mathrm{cm}^{-2}\right.$ $\left.\mathrm{s}^{-1}\right)$ in our sample. We detected the pulsar X-ray counterpart at $\left.\alpha_{\mathrm{X}}=21^{\mathrm{h}} 39^{\mathrm{m}} 56^{\mathrm{s}} .0( \pm 0.44) ; \delta_{\mathrm{X}}=47^{\circ} 16^{\prime} 13\right)^{\prime \prime} 0( \pm 0.52)$ with a significance of $5.5 \sigma$ (16 net source counts). As usual, from the pseudo-distance $D_{\gamma}=0.8 \mathrm{kpc}$ we derived $N_{\mathrm{H}}=10^{21} \mathrm{~cm}^{-2}$. By keeping it fixed, a fit with a PL with fixed photon index $\left(\Gamma_{\mathrm{X}}=2\right)$ gives an unabsorbed $\mathrm{X}$-ray flux $F_{\mathrm{X}}^{\mathrm{PL}}=$

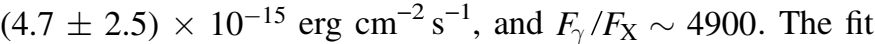
with a BB gives a very small emitting radius of only $33 \pm 9 \mathrm{~m}$, on a X-ray flux $F_{\mathrm{X}}^{\mathrm{BB}}=(2.7 \pm 1.4) \times 10^{-15} \mathrm{erg} \mathrm{cm}^{-2} \mathrm{~s}^{-1}$, and a $F_{\gamma} / F_{\mathrm{X}}>8500$.

\subsection{Optical and Infrared Observations}

None of the pulsars in our sample have been observed in the optical or infrared (IR, 2PC). For completeness, we also scanned optical/IR data from public imaging surveys. For PSR J1429-5911 we found serendipitous IR observations from the VVV (VISTA Variables in the Via Lactea; Emerson et al. 2006) survey, carried out at the ESO's Cerro Paranal Observatory (Chile) with the $4.1 \mathrm{~m}$ Visible and Infrared Survey Telescope for Astronomy (VISTA) and an IR camera (VIRCAM; Dalton et al. 2006). The fields of PSR J1838 -0537 and PSR J1846+0919 were serendipitously observed in the UKIDSS (UK Infrared Deep Sky Survey; Lawrence et al. 2007), carried out at the Mauna Kea Observatory (Hawaii) with the $3.8 \mathrm{~m}$ UKIRT and the Wide Field Camera (WFCAM; Casali et al. 2007). In all these cases, no objects are detected at the Chandra or XMM-Newton positions. The derived upper limits are orders of magnitude above the flux levels expected for the pulsar's age and spin-down power by scaling for the luminosity and distance of other pulsars detected in the IR (e.g., Mignani et al. 2012). Thus, these results are mainly to be considered as a reference for future deeper follow-up observations. Our $3 \sigma$ limits are $Z \gtrsim 21 ; Y \gtrsim$ $21 ; J \gtrsim 20 ; H \gtrsim 19 ; K \gtrsim 19$ (PSR J1429-5911), $J \sim 17$, $H \sim 19, K \sim 18$ (PSR J1838-0537), and $J \sim 20.5, H \sim 19.4$, $K \sim 18.6$ (PSR J1846+0919).

\section{DISCUSSION AND CONCLUSIONS}

Our Chandra and XMM-Newton observations yielded the identification of the X-ray counterparts of eight RQ LAT pulsars. Thus, the number of RQ LAT pulsars detected in $\mathrm{X}$-rays amounts now to 28 , with only 11 still missing an X-ray counterpart. This number has to be compared with the $30 \mathrm{RL}$ 


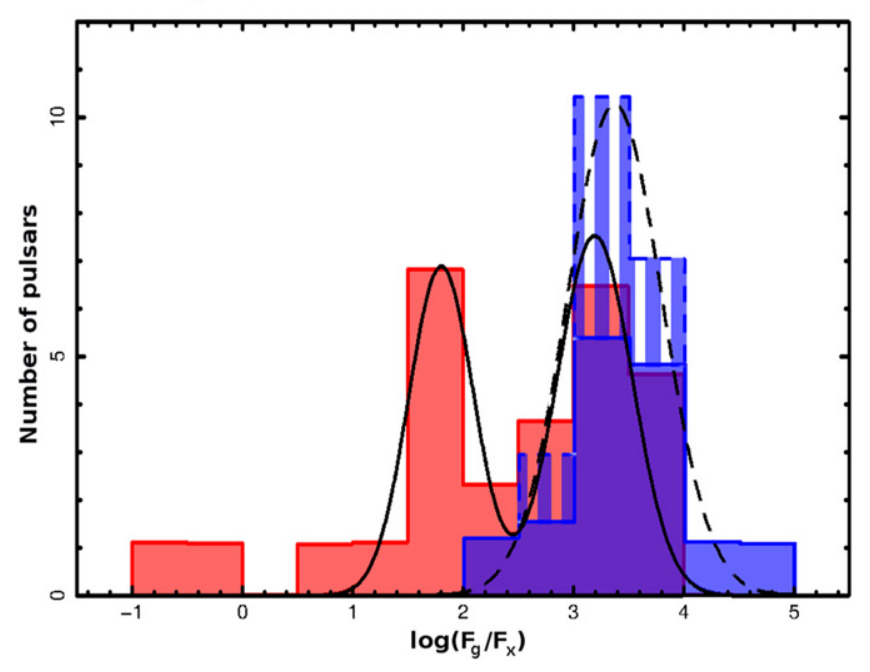

Figure 2. Histogram of the logarithm of the $\gamma$-to-X-ray flux ratio $\left(F_{\gamma} / F_{\mathrm{X}}\right)$ of LAT pulsars with high-confidence X-ray detections, as defined in Figure 3 of Marelli et al. (2011). Histograms for RL and RQ pulsars are shown in red and blue, respectively. The increment to the radio-quiet pulsar histogram for the eight pulsars that we detected in X-rays for the first time is shown in dashed blue and is added to the histogram of previously known radio-quiet pulsars. For these pulsars, we used the results of the PL fits to compute the $F_{X}$ values. The continuous and dashed lines report the best Gaussian fit for the RL and RQ distributions, respectively.

LAT pulsars (out of 42) detected in X-rays. Our enlarged X-ray database is important for a better understanding of the multiwavelength emission properties of LAT pulsars. In particular, focussing on the differences between young-to-middle-aged RQ and RL pulsars, Marelli et al. (2011) found that, for a given $\gamma$-ray flux, the former are intrinsically fainter in the X-ray band than the latter. Thus, the distance-independent $F_{\gamma} / F_{X}$ turned out to be a useful parameter to investigate the differences between the $\mathrm{X}$ - and $\gamma$-ray emission properties of the two pulsar families. For consistency, we followed the same approach as in Marelli et al. (2011) and we used our much enlarged sample of X-raydetected RQ pulsars to improve their analysis.

We note that millisecond pulsars (MS) were not included in the analysis of Marelli et al. (2011), owing to the fundamental differences between their magnetospheres and those of young/ middle-aged pulsars (e.g., Johnson et al. 2014). In fact, owing to their shorter periods, MS pulsars are thought to have a smaller magnetosphere than young pulsars, where the radius of the last closed field line is defined by the so-called lightcylinder radius $R_{\mathrm{LC}}=P c / 2 \pi$. Moreover, given their much longer and more complicated evolutionary history, the magnetic field topology of MS pulsars may be more complex than those of younger pulsars. This may in part explain why their pulse profiles are more intricate and diverse. Lastly, MS pulsars generally seem to be more massive than their younger counterparts. See Venter et al. (2014) for a more detailed discussion.

In addition to those obtained from our observations, we collected $\gamma$ - and X-ray best-fit spectra from the 2PC and papers published afterwards (PSR J1357-6429, Chang et al. 2012; J1741-2054, Marelli et al. 2014a; J1813-1246, Marelli et al. 2014b; J0357+3205, Marelli et al. 2013; J2055+2539, M. Marelli et al. 2015, in preparation). Figure 2 reports the histogram of the $\log \left(F_{\gamma} / F_{X}\right)$ values for all pulsars, both RL and $\mathrm{RQ}$, detected in X-rays and with a non-thermal X-ray spectrum. Improving on the method used in Marelli et al. (2011) and Marelli (2012), each pulsar has been represented with an asymmetric parabola, in order to account for the asymmetric errors on the $\mathrm{X}$ - and $\gamma$-ray fluxes. We separately highlighted in Figure 2 the $\log \left(F_{\gamma} / F_{X}\right)$ values of the eight pulsars with the newly discovered X-ray counterparts (Table 2), assuming a non-thermal X-ray spectrum for all of them. We fitted both RQ and RL pulsar distributions with single and double Gaussian models, taking into account Poissonian errors.

We confirmed that RQ pulsars are intrinsically fainter in $\mathrm{X}$-rays than the $\mathrm{RL}$ ones, with an average $\log \left(F_{\gamma} / F_{X}\right)$ of $3.38 \pm 0.10$ ( $1 \sigma$ error $)$ : at mean, RL pulsars have $F_{\gamma} / F_{X}$ values an order of magnitude lower (see later). The distribution of the $F_{\gamma} / F_{X}$ values for RQ pulsars is well-fitted by a Gaussian, with a null hypothesis probability of 0.62 , three degrees of freedom (dof), featuring a sharp peak (standard deviation of $0.43 \pm 0.09)$. This indicates very similar $X$ - and $\gamma$-ray emissions among the members of this family. The $F_{\gamma} / F_{X}$ values computed for the eight RQ pulsars with newly discovered counterparts (Table 2) are distributed around the peak, significantly increasing our statistics. We note that for four of the eight pulsars the X-ray spectrum could also be fitted by a single $\mathrm{BB}$ model, which would result in an even higher value of the corresponding $F_{\gamma} / F_{X}$.

As noted from Figure 2, the $F_{\gamma} / F_{X}$ values of RL pulsars are characterized by a more structured distribution. While a single Gaussian could fit the distribution, with an average $\log \left(F_{\gamma} / F_{X}\right)$ of $2.24 \pm 0.32$, the null hypothesis probability is found to be quite low (0.05, eight dof) and the distribution is much wider, with a standard deviation of $0.72 \pm 0.11$. By fitting the distribution with two Gaussians, we obtain two peaks at $1.81 \pm 0.11$ (standard deviation of $0.29 \pm 0.09$ ) and $3.20 \pm 0.14$ (standard deviation of $0.34 \pm 0.05$ ), with the better null hypothesis probability of 0.38 (five dof). An $f$-test (Bevington 1969) shows that the probability for a chance improvement is 0.03 , not enough to exclude the singleGaussian fit.

Interestingly enough, the second peak of the $F_{\gamma} / F_{X}$ distribution of RL pulsars would overlap the peak of the corresponding distribution for the RQ pulsars. If statistically confirmed, this might suggest that the separation between RL and RQ pulsars in the $F_{\gamma} / F_{X}$ space might not be as clear as was originally thought. At least partially, the overlap between the two peaks might be attributed to the subtle, and somehow arbitrary, distinction between RF and RQ pulsars. Indeed, some RL pulsars with the highest $F_{\gamma} / F_{X}$ values (e.g., J1741-2054 and $\mathrm{J} 1907+0602$, see Figure 3 in the 2PC) are radio-faint, whereas some RQ pulsars with the lowest $F_{\gamma} / F_{X}$ values, e.g., J1813-1246, are very distant and absorbed. Thus, they would be undetected if they had a radio luminosity comparable to those of J1741-2054 and J1907+0602. In any case, we found no clear correlation between the radio flux and the X-and $\gamma$-ray fluxes, which might have suggested that, e.g., radio-faint pulsars have higher $F_{\gamma} / F_{X}$ values than the radio-bright ones.

In a similar way, we built the histogram of the photon indices ratio, $\Gamma_{\gamma} / \Gamma_{X}$, for RL and RQ pulsar families (Figure 3). In both cases, the distributions are well fitted by a Gaussian (null hypothesis probabilities of 0.16 and $0.62,2$ dof for RL and RQ pulsars, respectively). Interestingly enough, the peaks of both distributions occur at very similar values of the $\Gamma_{\gamma} / \Gamma_{X}$ ratio, $-0.09 \pm 0.03$ and $-0.14 \pm 0.08$ for the $\mathrm{RL}$ and $\mathrm{RQ}$ pulsars, respectively. This suggests that the peculiar 


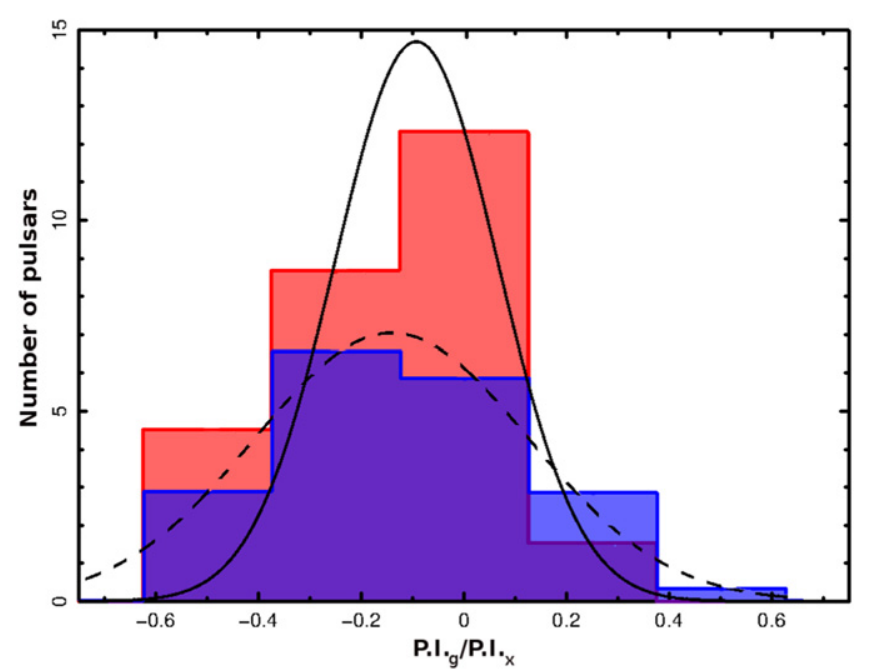

Figure 3. Histogram of the best fitted $\gamma$-to-X-ray photon index ratio $\left(\Gamma_{\gamma} / \Gamma_{\mathrm{X}}\right)$ of LAT pulsars with high-confidence X-ray detections, as defined in Figure 3 of Marelli et al. (2011). Histograms for RL and RQ pulsars are shown in red and blue, respectively. The pulsars for which the photon index has not been fitted are not included. The continuous and dashed lines report the best Gaussian fit for the RL and RQ distributions, respectively.

distribution seen in the $F_{\gamma} / F_{X}$ histogram (Figure 2) is not produced by an intrinsic difference between the spectral slopes of the two families. In the following, we explore some implications of the observed bimodal $F_{\gamma} / F_{X}$ distribution for RQ and RL pulsars on pulsar emission models.

Pulsar magnetospheric radiation is highly anisotropic and a complex antenna pattern (i.e., the directional dependence of the emitting power) results as the neutron star rotates and its beam sweeps the sky. In a specific energy range, different emission models expect different antenna patterns-the emission mechanism, geometry and luminosity depend on the magnetic field configuration, inclination angle and intensity, as well as on the pulsar period, but the physics ruling such mechanisms is not yet understood. For a complete discussion see e.g. Bai \& Spitkovsky (2010). Constraining the multi-wavelength antenna patterns as a function of different pulsar properties would yield crucial clues to understand pulsar magnetospheres.

The observed flux in a given energy range is a phaseaveraged cut through the antenna pattern for the Earth line of sight. A beaming factor $f_{\Omega}$ is usually defined as the ratio of the observed flux to the average flux over $4 \pi$ sr (e.g., Watters et al. 2009). In the radio range, the antenna pattern is usually described with the "cone plus core" heuristic model (e.g., Harding 2007). According to this model, then, the radio emission is centered on the magnetic axis (in a co-rotating frame) and radio loudness (or quietness) is merely a fortuitous effect, set by our line of sight intercepting (or missing) the radio antenna pattern of a pulsar, as a result of a small (or high) magnetic impact angle. In other words, RQ pulsars are simply those that are seen under a viewing angle substantially different from their magnetic inclination angle - the beaming factor for such lines of sight being essentially zero.

In the $\gamma$-ray range, recent works based on data collected by the Fermi-LAT (see e.g. Bai \& Spitkovsky 2010; Pierbattista et al. 2014) showed very complex antenna patterns, best described by outer magnetospheric models such as the Outer Gap (Romani 1996) or the Two-pole Caustic model
(Muslimov \& Harding 2004). According to such models, RQ pulsars should not have, on average, a different $\gamma$-ray beaming factor with respect to RL ones (e.g., Pierbattista et al. 2014indeed, beaming factors for RQ pulsars are expected to be more dispersed than for RL pulsars). On the other hand, although distance estimates for RQ pulsars are highly uncertain, there is no evidence for a larger luminosity (nor for a larger spin-down conversion efficiency $n_{\gamma} \equiv L_{\gamma} / \dot{E}$, as defined in Abdo et al. 2010) with respect to the RL ones, although some selection bias, related to highly pulsed $\gamma$-ray signal and little timing noise easing blind periodicity searches in $\gamma$-ray data, is certainly affecting our view of RQ pulsars.

In the above picture, it is tempting to link the different $F_{\gamma} / F_{X}$ distributions for RQ and RL pulsars to differences in their Xray emission properties, which could allow us to set first constraints on the poorly known X-ray antenna pattern. For the RQ pulsars (seen with large magnetic impact angle) the X-ray emission could possibly come from the outer magnetosphere, with an antenna pattern (and a beaming factor) possibly similar to the $\gamma$-ray one. This could explain the narrow $F_{\gamma} / F_{X}$ distribution for RQ pulsars in spite of their expected large dispersion in $\gamma$-ray beaming factors. Focusing on the RL pulsars, about half of the sample has $\log \left(F_{\gamma} / F_{\mathrm{X}}\right) \sim 1.8$, much lower than the average $\log \left(F_{\gamma} / F_{\mathrm{X}}\right) \sim 3.4$ for the RQ pulsars. This could suggest the existence of a luminous X-ray emission component that can only be seen for small magnetic impact angles, and thus possibly centered on the magnetic axis. Marelli et al. (2014b) explained the peculiar multi-wavelength behavior of the RQ pulsar J1813-1246 by modeling its X-ray emission using a polar cap model (Dyks et al. 2004): a lowaltitude cone beam with peak emission just inside the polar cap rim. The radio beam, centered on the X-ray cone, is missed by just a few degrees: a slightly different line of sight would have made J1813-1246 a bright radio pulsar. Such an X-ray polar cap emission component should display a large variability in luminosity and/or beaming factor within the RL pulsar sample in order to account for the large dispersion in $F_{\gamma} / F_{\mathrm{X}}$. Such a variability could be driven by other pulsar properties such as the spin-down-luminosity and/or the magnetic field configuration/intensity, as well as their evolution as a function of time.

A major step forward in our understanding of the overall multi-wavelength emission geometry of pulsars could be obtained by performing simultaneous fitting of their multiwavelength light curves. Recently, Pierbattista et al. (2014) have jointly fit $\gamma$-ray and radio light curves with simulated $\gamma$ ray and radio emission patterns. This allowed them to investigate some relations between observable characteristics and intrinsic pulsar parameters, encouraging the creation and testing of new models (see e.g. Kalapotharakos 2012; Li et al. 2012). Also owing to the increasing number of RQ pulsars, the above analysis should be extended to include X-ray light curves, taking into account both thermal and non-thermal pulsed emission. Simultaneous fitting and phase-resolved spectral analysis of multi-wavelength emission of pulsars will allow us to test different emission models and to build new models for the overall emission, also better explaining the results presented in this paper. Unfortunately, of the $28 \mathrm{RQ}$ LAT pulsars now detected in X-rays, only seven have been observed for a sufficiently long integration time to detect X-ray pulsations. Similarly, of the 30 RL LAT pulsars with an X-ray counterpart, only 15 are known to pulsate in the X-ray band. Therefore, an important contribution to the theoretical analysis 
would come from the detection of X-ray pulsations for most of the brightest pulsars through deep follow-up XMM-Newton and Chandra observations. While a number of much brighter RQ and RL pulsars are better suited for such a future study, deep observations of XMM-Newton, the best in-flight telescope to perform such a search, could allow us to detect pulsations from our eight pulsars. For instance, if we assume a Lorentzian light curve with a duty cycle of 0.1 , with an entire orbit of XMM observation $(130 \mathrm{ks})$ we would detect pulsations at $5 \sigma$ in case of a pulsed fraction of $\sim 40 \%$ for the brightest of our eight pulsars to $100 \%$ for the weakest one.

We thank Andrea Belfiore ("Mario") for the useful discussions on the $\gamma$-ray timing positions of LAT pulsars. The research leading to these results has received funding from the European Commission Seventh Framework Programme (FP7/2007-2013) under grant agreement n. 267251. This work was supported by the ASI-INAF contract I/037/12/0, art.22 L.240/2010 for the project "Calibrazione ed Analisi del satallite NuSTAR." Support for this work was provided by the National Aeronautics and Space Administration through Chandra Award Numbers GO2-13093X and GO3-14075X issued by the Chandra X-ray Observatory Center, which is operated by the Smithsonian Astrophysical Observatory for and on behalf of the National Aeronautics and Space Administration under contract NAS8-03060.

Facilities: XMM, CXO, ESO:VISTA, UKIRT

\section{REFERENCES}

Abdo, A. A., Ackermann, M., Ajello, M., et al. 2010, ApJS, 187, 460

Abdo, A. A., Ajello, M., Allafort, A., et al. 2013, ApJS, 208, 17

Atwood, W. B., Abdo, A. A., Ackermann, M., et al. 2009, ApJ, 697, 1071

Bai, X., \& Spitkovsky, A. 2010, ApJ, 715, 1282

Bevington, P. R. 1969, drea.book

Boschin, W. 2002, A\&A, 396, 397
Caraveo, P. A. 2014, ARA\&A, 52, 211

Casali, M., Adamson, A., Alves de Oliveira, C., et al. 2007, A\&A, 467, 777 Cash, W. 1979, ApJ, 228, 939

Chang, C., Pavlov, G. G., Kargaltsev, O., \& Shibanov, Y. A. 2012, ApJ, 744,81

Cheng, K. S., Ho, C., \& Ruderman, M. 1986, ApJ, 300, 500

Cordes, J. M., \& Lazio, T. J. W. 2002, arXiv:0207156

Dalton, G. B., Caldwell, M., Ward, A. K., et al. 2006, SPIE, 6269, 0

De Luca, A., Caraveo, P. A., Mereghetti, S., Negroni, M., \& Bignami, G. F. 2005, ApJ, 623, 1051

De Luca, A., Mignani, R. P., Marelli, M., et al. 2013, ApJ, 765, 19

Dyks, J., Harding, A. K., \& Rudak, B. 2004, ApJ, 606, 1125

Emerson, J., Irwin, M., \& Hambly, N. 2006, SPIE, 6270, 0

Harding, A. K. 2007, arXiv:0710.3517

Harding, A. K., \& Muslimov, A. G. 2001, ApJ, 556, 987

Harding, A. K., \& Muslimov, A. G. 2004, cosp, 35, 562

Johnson, T. J., Venter, C., Harding, A. K., et al. 2014, ApJS, 213, 6

Kalapotharakos, C. 2012, ApJ, 754, 1

Lawrence, A., Warren, S. J., Almaini, O., et al. 2007, MNRAS, 379, 1599

Li, J., Spitkovsky, A., \& Tchekhovskoy, A. 2012, ApJ, 746, 60

Marelli, M. 2012, arXiv:1205.1748

Marelli, M., Belfiore, A., Saz Parkinson, P., et al. 2014a, ApJ, 790, 51

Marelli, M., de Luca, A., \& Caraveo, P. A. 2011, ApJ, 733, 82

Marelli, M., de Luca, A., Salvetti, D., et al. 2013, ApJ, 765, 36

Marelli, M., Harding, A., Pizzocaro, D., et al. 2014b, ApJ, 795, 168

Mignani, R. P., Razzano, M., Esposito, P., et al. 2012, A\&A, 543, 130

Muslimov, A. G., \& Harding, A. K. 2004, ApJ, 606, 1143

Novara, G., La Palombara, N., Mignani, R. P., et al. 2009, A\&A, 501, 103

Pierbattista, M., Harding, A. K., Grenier, I. A., et al. 2014, arXiv:1403.3849

Pletsch, H. J., Guillemot, L., Allen, B., et al. 2012a, ApJ, 755, 20

Pletsch, H. J., Guillemot, L., Allen, B., et al. 2012b, ApJ, 744, 105

Ray, P. S., Kerr, M., Parent, D., et al. 2011, ApJS, 194, 17

Romani, R. W. 1996, ApJ, 470, 469

Saz Parkinson, P. M. 2011, arXiv:1101.3096

Saz Parkinson, P. M., Dormody, M., Ziegler, M., et al. 2010, ApJ, 725, 571

Schlafly, E. F., \& Finkbeiner, D. P. 2011, ApJ, 737, 103

Schlegel, D. J., Finkbeiner, D. P., \& Davis, M. 1998, ApJ, 500, 525

Uchiyama, Y., Maeda, Y., Ebara, M., et al. 2008, PASJ, 60, 35

Venter, C., Johnson, T. J., Harding, A. K., \& Grove, J. E. 2014 arXiv: 1411.0559

Watters, K. P., Romani, R. W., Weltevrede, P., \& Johnston, S. 2009, ApJ, 695,1298 